\title{
Periodic Solutions of Some Infinite-Dimensional Hamiltonian Systems Associated with Non-Linear Partial Difference Equations. II
}

\author{
Claudio Albanese ${ }^{1, \star}$, Jürg Fröhlich ${ }^{1}$, and Thomas Spencer ${ }^{2}$ \\ 1 Theoretical Physics, ETH-Hönggerberg, CH-8093 Zürich, Switzerland \\ 2 The Institute for Advanced Study, Princeton, NJ 08540, USA
}

\begin{abstract}
This is our second paper devoted to the study of some non-linear Schrödinger equations with random potential. We study the non-linear eigenvalue problems corresponding to these equations. We exhibit a countable family of eigenfunctions corresponding to simple eigenvalues densely embedded in the "band tails." Contrary to our results in the first paper, the results established in the present paper hold for an arbitrary strength of the non-linear (cubic) term in the non-linear Schrödinger equation.
\end{abstract}

\section{Contents}

Part II - Proof of Theorem NL2 . . . . . . . . . . . . . . . . . . . . 677

Sect. 5 - Introduction . . . . . . . . . . . . . . . . . . . . . . . . 677

Sect. 6 - Strategy for the Proof of the Theorem NL2 . . . . . . . . . . . . . 678

Sect. 7 - The First Step . . . . . . . . . . . . . . . . . . . . . . . . 682

Sect. 8 - Construction of $\Omega_{n+1}$ and $B_{n+1}$. . . . . . . . . . . . . . . . . . . . 684

Sect. 9 - Construction of $\phi_{n+1}$. . . . . . . . . . . . . . . . . . . . . . . 686

Sect. 10 - Construction of $\psi_{n+1}$. The Proof Completed . . . . . . . . . . . . . 690

\section{Introduction}

In this paper we continue our analysis of the non-linear eigenvalue problem

$$
\left.\begin{array}{l}
(-\Delta+V(x)+\lambda W(u)(x)) u(x)=E u(x) \\
u \in \ell^{2}\left(\mathbb{Z}^{v}\right), \quad\|u\|_{2}=1,
\end{array}\right\}
$$

which was initiated in [1]. In (5.1), $\Delta$ is the finite difference Laplacian,

$$
(\Delta u)(x)=\sum_{y:|y-x|=1} u(y)
$$

\footnotetext{
* Address after July, 1988: Department of Mathematics, University of California, Los Angeles, CA 90024, USA
} 
$V(x)$ are i.i.d. random variables with distribution

$$
d \varrho(V)=\sqrt{\frac{1}{\pi \zeta}} e^{-V^{2} / \zeta} d V,
$$

and $W(u)(x)$ is defined as follows:

$$
W(u)(x)=\sum_{y} W(|y-x|)|u(y)|^{2} .
$$

We assume that the function $W(|x|)$ decays exponentially fast with rate $m_{W}>0$. To normalize $W$, we impose the following condition:

$$
W(|y-x|) \leqq e^{-m_{W}|y-x|} .
$$

In the first paper [1], we considered the problem of perturbing the solution of the linear problem (5.1) corresponding to $\lambda=0$. We found that this is possible, provided that $\lambda$ is restricted to a set, $\Lambda$, which is not an interval, and we showed that the set of eigenvalues one finds for $\lambda \in \Lambda$ is a (random) Cantor set. In this paper, we look at the non-linear eigenvalue problem (5.1) from a different perspective: We fix some $\lambda>0$ and propose to construct solutions of (5.1), for the given value of $\lambda$. Our main result is the following theorem:

Theorem NL2. Consider the distribution (5.3), and suppose that the kernel $W(|x-y|)$ of the operator $W$ decays exponentially, with rate $m_{W}>0$. Then there exists a constant $E_{1}^{\prime}(v, \zeta)$ and, for every $\lambda>0$, a set, $\Omega(\lambda)$, of potentials, $V$, of full measure with the property that, for $V \in \Omega(\lambda)$, the non-linear Schrödinger equation (5.1) has infinitely many solutions, and the corresponding eigenvalues form a dense subset of

$$
\left\{E:|E|>E_{1}^{\prime}(v, \zeta)\right\} \text {. }
$$

We remark that in [1] we stated this theorem under the more restrictive assumption that $W(|x|)$ have finite range. However, this turns out to be easily avoidable.

This paper is fairly technical and many notations are required. Our most important notations are summarized in Appendix D.

\section{Strategy for the Proof of Theorem NL2}

To explain our approach to this problem, let us fix an interval $(\widetilde{E}-\varepsilon, \widetilde{E}+\varepsilon)$, with $\varepsilon>0$ and with $|\widetilde{E}|$ large enough. What we must prove is that, for every $\lambda>0$, the set $\Omega(\lambda, \widetilde{E}, \varepsilon)$ of potentials, $V$, for which the non-linear eigenvalue problem (5.1) has a solution $(E, \psi)$, with $E \in(\widetilde{E}-\varepsilon, \widetilde{E}+\varepsilon)$, has full measure. Noticing that the set $\Omega(\lambda, \widetilde{E}, \varepsilon)$ is invariant under translations and that the group of translations acts ergodically, we see that it is enough to prove that $\Omega(\lambda, \widetilde{E}, \varepsilon)$ has positive measure. Our proof consists in an explicit construction of a subset, $\Omega_{\infty}$, of $\Omega(\lambda, \widetilde{E}, \varepsilon)$ of positive measure.

Let us fix an origin $\underline{0}$ in the lattice $\mathbb{Z}^{v}$. Let $d_{0}>0$ be a number to be chosen later on, and let us consider the following family of blocks:

$$
B_{n}^{d}=\left\{|x| \leqq d_{0}^{(5 / 4)^{n}} \equiv d_{n}\right\} \text {. }
$$


We shall define $\Omega_{\infty}$ as a set of potentials $V$ for which there is a sequence $B_{n}$ of blocks close to $B_{n}^{d}$ such that the eigenvalue problems

$$
\left.\begin{array}{l}
-\Delta \psi_{n}+V \psi_{n}+\lambda W\left(\psi_{n}\right) \psi_{n}=E_{n} \psi_{n} \\
\psi_{n} \in \ell^{2}\left(B_{n}\right), \quad\left\|\psi_{n}\right\|_{2}=1,\left.\quad \psi_{n}\right|_{\partial B_{n}}=0,
\end{array}\right\}
$$

have solutions $\left(E_{n}, \psi_{n}\right)$ converging, as $n \uparrow \infty$, to a solution $(E, \psi)$ of $(5.1)$, with $E \in\left(E_{0}-\frac{\varepsilon}{2}, E_{0}+\frac{\varepsilon}{2}\right)$, where $E_{0}$ is the eigenvalue of (6.2) for $n=0$. The set $\Omega_{\infty}$ will be constructed as the intersection

$$
\Omega_{\infty}=\bigcap_{n=0}^{\infty} \Omega_{n}
$$

of a decreasing family of subsets

$$
\Omega_{0} \supset \Omega_{1} \supset \Omega_{2} \supset \ldots
$$

Our construction is inductive and, at each iteration step, we find a quadruple $\left(\Omega_{n}, B_{n}, E_{n}, \psi_{n}\right)$. To pass from the $n^{\text {th }}$ step to the $(n+1)^{\text {st }}$ step (for $n \geqq 1$ ), we need to know some properties of the quadruples $\left(\Omega_{k}, B_{k}, E_{k}, \psi_{k}\right)$, with $k \leqq n$. These properties are summarized in an induction hypothesis, $\mathscr{I}(k)$, to be proven inductively, which will be introduced later on in this section.

Before describing the induction hypothesis, we introduce some other piece of notation. Let $\left(\Omega_{n}, B_{n}, E_{n}, \psi_{n}\right)$ be one of the quadruples described above. In the following, we shall regard $\psi_{n}$ as a function defined on the entire lattice $\mathbb{Z}^{v}$, by defining it to be zero outside $B_{n}$. Let $H_{\lambda, n}^{V}$ denote the operator

$$
-\Delta+V+\lambda W\left(\psi_{n}\right) \text {. }
$$

The operator (6.5), with $\lambda=0$, will be denoted by $H_{0}^{V}$. If $H$ is a Schrödinger operator and $A$ is a subset of $\mathbb{Z}^{v}$, let $H(A)$ denote the restriction of $H$ to $\ell^{2}(A)$ with Dirichlet boundary conditions. In the following we make use of the Green functions:

$$
\begin{aligned}
G_{A}^{\lambda, n}(z) & \equiv\left(z-H_{\lambda, n}^{V}(A)\right)^{-1}, \\
G_{A}^{0}(z) & \equiv\left(z-H_{0}^{V}(A)\right)^{-1} ;
\end{aligned}
$$

$\delta_{n}$ denotes the number $\exp \left(-\sqrt{d_{n}}\right)$, with $d_{n}$ given in $(6.1), \mathscr{C}_{n}$ the circle

$$
\mathscr{C}_{n}=\left\{z \in \mathbb{C}:\left|z-E_{n}\right|=\frac{1}{2} \delta_{n}\right\},
$$

and $\overline{\mathscr{C}}_{n}$ the disc enclosed by $\mathscr{C}_{n}$. Finally, we set $A_{n}^{d} \equiv B_{n}^{d} \backslash B_{n-1}^{d}$, and $A_{n} \equiv B_{n} \backslash B_{n-1}$.

The construction of the first quadruple $\left(\Omega_{0}, B_{0}, E_{0}, \psi_{0}\right)$ is somewhat special and proceeds as follows: We set $B_{0} \equiv B_{0}^{d}$.

Definition of $\Omega_{0} . \Omega_{0}$ is contained in the (positive measure) set of potentials, $V$, which have a single well of depth at least $U$ at the origin $\underline{0} \in \mathbb{Z}^{v}$, i.e. we have

$$
0<U<\inf _{x \in B_{1}^{d} \backslash\{0\}}(V(x)-V(0)), \quad \forall V \in \Omega_{0} .
$$

In the following we shall specify the minimal size, $U^{*}(\lambda)$, that $U$ must have. Moreover, we require that the problem (6.2), for $n=0$, has a solution with 
eigenvalue $E_{0} \in\left[\widetilde{E}-\frac{1}{2} \varepsilon, \widetilde{E}+\frac{1}{2} \varepsilon\right] .\left(E_{0}, \psi_{0}\right)$ is constructed as the solution of (6.2), for $n=0$, on the bifurcation branch emanating from the ground state solution of the linear problem (6.2), with $n=\lambda=0$.

The discrete version of a global bifurcation theorem, due to Rabinowitz [2], assures one that this is possible, for all $\lambda>0$. Moreover, $B_{1}$ will be chosen to be $B_{1}^{d}$, and $\left(\Omega_{1}, E_{1}, \psi_{1}\right)$ will be constructed similarly to $\Omega_{0}, E_{0}, \psi_{0}$. The details are given in Sect. 7. An alternative construction of a suitable subset, $\Omega_{0}$, of potentials from which our construction can proceed is given in Appendix A.

For $n \geqq 2$, we shall construct solutions of (6.2) with the help of a two-stepprocedure: First, we consider the spectral problem for the linear operator $H_{\lambda, n}^{V}\left(B_{n}\right)$ defined by (6.5), where $B_{n}$ is a set close to $B_{n}^{d}$. The set $\Omega_{n}$ is defined as the subset of $\Omega_{n-1}$ which fulfills a suitable condition on the spectrum of the operator $H_{0}^{V}\left(A_{n}^{d} \cup A_{n+1}^{d}\right)$. On one hand, our condition will guarantee that the linear eigenvalue problem

$$
\left.\begin{array}{l}
H_{\lambda, n-1}^{V}\left(B_{n}\right) \phi_{n}=E_{n}^{\ell} \phi_{n} \\
\phi_{n} \in \ell^{2}\left(B_{n}\right), \quad\left\|\phi_{n}\right\|_{2}=1
\end{array}\right\}
$$

has a solution $\left(E_{n}^{\ell}, \phi_{n}\right)$ close to $\left(E_{n-1}, \psi_{n-1}\right)$. On the other hand, it will guarantee that $\Omega_{\infty} \equiv \bigcap_{n=0}^{\infty} \Omega_{n}$ has positive measure.

Second, we consider the following family of eigenvalue problems connecting (6.10) with (6.2):

$$
\begin{aligned}
& \left.\left\{H_{\lambda, n-1}^{V}\left(B_{n}\right)+\beta\left[W\left(u_{\beta}\right)-W\left(\psi_{n-1}\right)\right]\right\} u_{\beta}=E_{\beta} u_{\beta}\right\} \\
& u_{\beta} \in \ell^{2}\left(B_{n}\right), \quad\left\|u_{\beta}\right\|_{2}=1 .
\end{aligned}
$$

Here $\beta$ is a parameter ranging over $[0, \lambda]$, and we look for a smooth curve, $\left(E_{\beta}, u_{\beta}\right)$, of solutions of (6.11) such that

$$
\left.\left(E_{\beta}, u_{\beta}\right)\right|_{\beta=0}=\left(E_{n}^{\ell}, \phi_{n}\right) .
$$

We then set $\left(E_{n}, \psi_{n}\right)=\left(E_{\lambda}, u_{\lambda}\right)$. See [3] and [4] for other applications of this method to the construction of solutions of non-linear eigenvalue problems.

We are now ready to describe our induction hypothesis, $\mathscr{I}(n)$, for $n \geqq 1$. It consists of the following statements:

$\mathscr{I}_{1}(n): \Omega_{n} \subseteq \Omega_{n-1}$, and if $n \geqq 2$,

$$
\mu\left(\Omega_{n-1} \backslash \Omega_{n}\right) \leqq \varrho\left(E_{n-1}, \zeta\right) d_{n-2}^{-1},
$$

where $\varrho(E, \zeta) \downarrow 0$, as $|E|+\zeta \uparrow \infty$.

$$
\mathscr{I}_{2}(n): \quad B_{n}^{d} \subset B_{n} \subset B_{n+1}^{d},
$$

and

$$
\frac{1}{2} d_{n-1} \leqq \operatorname{dist}\left(x, \gamma_{n}\right) \leqq d_{n-1}, \quad \forall x \in \gamma_{n}^{d},
$$

where $\gamma_{n}=\partial B_{n}, \gamma_{n}^{d}=\partial B_{n}^{d}$.

$\mathscr{I}_{3}(n)$ : If $A_{k}=B_{k} \backslash B_{k-1}$, then, for a certain $m_{0}>0$ and for all $k \leqq n$, we have $\operatorname{dist}\left(E_{n}, \sigma\left(H_{\lambda, n-1}^{V}\left(A_{k}\right)\right)\right) \geqq \frac{1}{2} \delta_{k-1}$, and

$$
\left|G_{A_{k}}^{\lambda, n-1}(x, y ; z)\right| \leqq e^{-m_{0}|x-y|},
$$


provided $\left|z-E_{n}\right| \leqq \frac{1}{2} \delta_{k}$ and $|x-y| \geqq \frac{1}{5} d_{k-1}$

$$
\mathscr{I}_{4}(n): \quad\left|E_{n}^{\ell}-E_{n-1}\right| \leqq \frac{1}{2} \exp \left(-\frac{1}{2} m d_{n-1}\right),
$$

and

$$
\left|E_{n}-E_{n}^{\ell}\right| \leqq \frac{1}{2} \exp \left(-\frac{1}{2} m d_{n-1}\right)
$$

where $m=\min \left(m_{0}, m_{W}\right)$.

$$
\begin{array}{ll}
\mathscr{I}_{5}(n): & \operatorname{dist}\left(E_{n-1}, \sigma\left(H_{\lambda, n-1}^{V}\left(B_{n}\right)\right) \backslash\left\{E_{n}^{\ell}\right\}\right) \geqq \frac{1}{4} \delta_{n-1} . \\
\mathscr{I}_{6}(n): & \left\|\phi_{n}-\psi_{n-1}\right\|_{\infty} \leqq \frac{1}{2} \min \left(1, \lambda^{-1}\right) e^{-\frac{1}{2} m d_{n-1}},
\end{array}
$$

and

$$
\begin{aligned}
& \left\|\psi_{n}-\phi_{n}\right\|_{\infty} \leqq \frac{1}{2} \min \left(1, \lambda^{-1}\right) e^{-\frac{1}{2} m d_{n-1}} . \\
\mathscr{I}_{7}(n): & \left|\phi_{n}(x)\right| \leqq 2 v e^{-m|x|}, \quad \forall|x| \geqq \frac{1}{5} d_{n},
\end{aligned}
$$

and

$$
\left|\psi_{n}(x)\right| \leqq 2 v e^{-m|x|}, \quad \forall|x| \geqq \frac{1}{5} d_{n}
$$

( $v$ is the dimension of the lattice).

To conclude this section, we state a theorem that will be proven in the next sections and from which Theorem NL2 follows.

Theorem 6.1. If $|\widetilde{E}|$ or $\zeta$ are large enough, then, for all choices of an origin $\underline{0} \in \mathbb{Z}^{v}$, all $\varepsilon>0$ and all $\lambda>0$, there are constants $d_{0}^{*}(\lambda, \varepsilon)$ and $U_{0}^{*}(\lambda)$ such that if $d_{0}>d_{0}^{*}(\lambda, \varepsilon)$, $U>U_{0}^{*}(\lambda)$ and for all $V \in \Omega_{0}$ (where $\Omega_{0}$ has been defined in (6.9)), there is a sequence of quadruples $\left\{\left(\Omega_{n}, B_{n}, E_{n}, \psi_{n}\right)\right\}_{n \geqq 0}$ satisfying the induction hypothesis $\mathscr{I}(n)$, for all $n$, and such that

$$
\sum_{n=0}^{\infty}\left|E_{n+1}-E_{n}\right|<\frac{\varepsilon}{2}
$$

We note that if Theorem 6.1 holds, then the sequence $\left\{\left(E_{n}, \psi_{n}\right)\right\}_{n \geqq 0}$ converges in the $\mathbb{R} \times \ell^{2}\left(\mathbb{Z}^{v}\right)$-norm. In fact, thanks to $\mathscr{I}_{6}(n)$, we have

$$
\left\|\psi_{n}-\psi_{n-1}\right\|_{2} \leqq \min \left(1, \lambda^{-1}\right)\left|B_{n}\right| \exp \left(-\frac{1}{2} m d_{n-1}\right),
$$

and the sequence $\left|B_{n}\right| \exp \left(-\frac{1}{2} m d_{n-1}\right)$ converges to zero, as $n \uparrow \infty$. Moreover, we have

$$
\begin{aligned}
\mu\left(\Omega_{\infty}\right) & =\mu\left(\bigcap_{n=0}^{\infty} \Omega_{n}\right) \geqq \mu\left(\Omega_{0}\right)-\sum_{k=0}^{\infty} \mu\left(\Omega_{n} \backslash \Omega_{n+1}\right) \\
& \geqq \mu\left(\Omega_{0}\right)-\left(\sup _{E \in[\tilde{E}-\varepsilon, \tilde{E}+\varepsilon]} \bar{\varrho}(E, \zeta)\right) \sum_{n=0}^{\infty} d_{n}^{-1} .
\end{aligned}
$$

For $|\widetilde{E}|+\zeta$ large enough, we conclude that $\mu\left(\Omega_{\infty}\right)>0$. This and the ergodicity of the action of the translation group prove Theorem NL2. 


\section{The First Step}

In this section we discuss the construction of $\left(\Omega_{n}, B_{n}, E_{n}, \psi_{n}\right)$ for $n=0,1$ and of $\left(E_{1}^{\ell}, \phi_{1}^{\ell}\right)$. We also prove some lemmas which, together with the methods explained in Sects. 9 and 10, imply that $\mathscr{I}(1)$ holds. Our aim being to prove Theorem 6.1, it is enough to prove our statements for $d_{0}$ and $U$ large enough.

We set $B_{0}=B_{0}^{d}, B_{1}=B_{1}^{d}$ and choose $\Omega_{0}=\Omega_{1}$ to be the set of potentials such that

$$
0<U<\inf _{x \in B_{1}^{d} \backslash\{0\}} V(x)-V(0),
$$

and such that the problem

$$
\left.\begin{array}{l}
-\Delta \psi_{0}+V \psi_{0}+\lambda W\left(\psi_{0}\right) \psi_{0}=E_{0} \psi_{0} \\
\psi_{0} \in \ell^{2}\left(B_{0}^{d}\right), \quad\left\|\psi_{0}\right\|_{2}=1,\left.\quad \psi_{0}\right|_{\partial B_{0}^{d}}=0,
\end{array}\right\}
$$

has a positive solution with

$$
E_{0} \in\left[\widetilde{E}-\frac{\varepsilon}{2}, \widetilde{E}+\frac{\varepsilon}{2}\right] .
$$

To prove that our choice of $\Omega_{0}$ is consistent, we need the following extension of a global bifurcation theorem due to Rabinowitz [2]:

Lemma 7.1. Problem (7.2) admits a solution $\left(\psi_{0}, E_{0}\right)$, with $\psi_{0} \geqq 0$, for all $\lambda \geqq 0, d_{0}>0$, and $V$ as in (7.1).

Proof. From the general theory of bifurcation off nondegenerate eigensolutions for finite dimensional problems [8], we know that there exists a curve $\gamma: \alpha \mapsto\left(u_{\alpha}, E_{\alpha}, \lambda_{\alpha}\right), \alpha \in[0,1)$, in the space $\ell^{2}\left(B_{0}\right) \times \mathbb{R}_{E} \times \mathbb{R}_{\lambda}$ such that $\left(u_{\alpha}, E_{\alpha}\right)$ solves (7.2) with $\lambda=\lambda_{\alpha}$. The curve $\gamma$ is differentiable, for $\alpha \in[0,1)$, with initial condition $\left(u_{0}, E_{0}, \lambda_{0}\right)=\left(u_{0}, E_{0}^{\ell}, 0\right)$, and either

(a) the projection of $\gamma$ onto the subspace $\mathbb{R}_{E} \times \mathbb{R}_{\lambda}$ is unbounded; or

(b) we have

$$
\lim _{\alpha \uparrow 1}\left(u_{\alpha}, E_{\alpha}, \lambda_{\alpha}\right)=\left(u_{1}, E_{1}, 0\right),
$$

where $\left(u_{1}, E_{1}\right) \neq\left(u_{0}, E_{0}\right)$; see Fig. 1. In our situation, (b) cannot occur, for the following reason: An excited state of the Hamiltonian $(-\Delta+V)\left(B_{0}\right)$ cannot be everywhere non-negative, because otherwise it would not be orthogonal to $u_{n}$.

Fig. 1

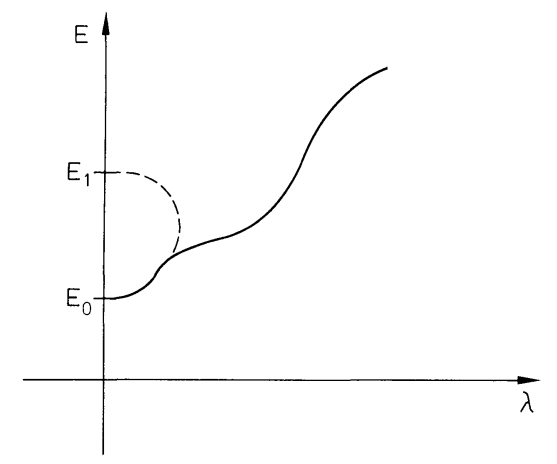


Hence, if (b) held there would be a point $(\bar{u}, \bar{E}, \bar{\lambda})$ on $\gamma$ with $\bar{u} \geqq 0$ and $\bar{u}(x)=0$, for every $x$ in a non-empty set $Q \subset B_{0} \backslash \partial B_{0}$.

But if $Q \neq \emptyset$ then $Q=B_{0}$. In fact, if $x_{0} \in Q$ then $(\Delta u)\left(x_{0}\right)=0$ and this implies that $u(x)$ is zero also on the sites $x$ neighbouring $x_{0}$. Since $B_{0}$ is connected, we conclude that $Q=B_{0}$, which contradicts (b) above.

Thus in our situation, (a) holds. Clearly, $\gamma$ cannot be unbounded in the direction of $\mathbb{R}_{E}$ without being unbounded in the direction $\mathbb{R}_{\lambda}$ as well. This means that (7.2) admits a positive solution for arbitrarily large $\lambda$. Q.E.D.

Remark. See Appendix A for an alternative choice of $\Omega_{0}$.

The next few lemmas illustrate some of the properties of $\psi_{0}$ which hold if $U$ is large. [Recall that $\psi_{0}$ is a solution of (7.2).]

Lemma 7.2. We have

$$
\operatorname{dist}\left(E_{0}, \sigma\left(H_{\lambda, 0}^{V}\left(B_{0}\right)\right) \backslash\left\{E_{0}\right\}\right) \geqq-6 v-\lambda+U\left[2-\psi_{0}(0)^{-2}\right]
$$

and $\psi_{0}(0)^{2} \uparrow 1$, as $U \uparrow \infty$.

Proof. $\psi_{0}$ is the ground state of the Hamiltonian $H_{\lambda, 0}^{V}\left(B_{0}\right)$, corresponding to the eigenvalue $E_{0}$. Let $\chi(x)$ be the first excited state of $H_{\lambda, 0}^{V}\left(B_{0}\right)$ and $E^{\prime}$ its energy. The orthogonality relation

$$
\sum_{x} \chi(x) \psi_{0}(x)=0
$$

gives

$$
\chi(0) \psi_{0}(0)=-\sum_{x \neq 0} \chi(x) \psi_{0}(x)
$$

whence

$$
\chi(0)^{2}=\psi_{0}(0)^{-2}\left|\sum_{x \neq 0} \chi(x) \psi_{0}(x)\right|^{2} \leqq \psi_{0}(0)^{-2} \sum_{x \neq 0} \psi_{0}(x)^{2}=\psi_{0}(0)^{-2}-1 .
$$

This bound and some simple inequalities yield

$$
\begin{aligned}
E^{\prime}-E_{0}= & \sum_{x}\left[-\chi(x)(\Delta \chi)(x)+\psi_{0}(x)\left(\Delta \psi_{0}\right)(x)+\lambda \psi_{0}(x)^{2}\left(\chi(x)^{2}-\psi_{0}(x)^{2}\right)\right] \\
& +\sum_{x \neq 0} V(x)\left(\chi(x)^{2}-\psi_{0}(x)^{2}\right)+V(0)\left(\chi(0)^{2}-\psi_{0}(0)^{2}\right) \\
\geqq & 4 v-\lambda+U\left[2-\psi_{0}(0)^{-2}\right]+V(0)-(2-V(0)) . \quad \text { Q.E.D. }
\end{aligned}
$$

Lemma 7.3. We have

$$
\gamma \equiv \inf _{x \in B_{0} \backslash\{0\}} V(x)-E_{0} \geqq U-(\lambda+2 v)
$$

Proof. Since $\psi_{0}(x)>0, \psi_{0}(x)$ is the ground state of $H_{\lambda, 0}^{V}\left(B_{0}\right)$, and thus we have

$$
\begin{aligned}
E_{0} & =\inf _{\substack{\|\psi\|_{2}=\left.1 \\
\psi\right|_{i B_{0}}=0}} \sum_{x \in B_{0}}\left[-\psi(x)(\Delta \psi)(x)+V(x) \psi(x)^{2}+\lambda \psi_{0}(x)^{2} \psi(x)^{2}\right] \\
& \leqq \lambda+2 v+\inf _{x \in B_{0} \backslash\{0\}} V(x)-U,
\end{aligned}
$$

whence (7.5) follows. Q.E.D. 
Lemma 7.4. If $\gamma>2 v$, then for all $\varepsilon \in(0, \gamma)$ we have

$$
\left|\psi_{0}(x)\right| \leqq 2 v \varepsilon^{-1} \exp (-m(\gamma, \varepsilon)|x|),
$$

where $m(\gamma, \varepsilon)=\ln \left(1+\frac{1}{2 v}(\gamma-\varepsilon-2 v)\right)$.

This bound can be obtained with the help of a Combes-Thomas argument that will be sketched in Appendix C. From the two lemmas above one can derive the following corollary:

Corollary. There is a constant $m_{0}(U)$ depending on $U$ and diverging to $\infty$, as $U \uparrow \infty$, such that

$$
\left|\psi_{0}(x)\right| \leqq e^{-m_{0}(U)|x|}, \quad \forall x \in B_{0} .
$$

The quadruple $\left(\Omega_{1}, B_{1}, E_{1}, \psi_{1}\right)$ will be defined in a completely analogous way. Namely, we set $B_{1}=B_{1}^{d}$, we define $\Omega_{1}$ as the set of potentials, $V$, satisfying the condition (7.1) and we define $\left(E_{1}, \psi_{1}\right)$ to be a solution of the problem (7.2) with Dirichlet boundary conditions on $\partial B_{1}$ instead that on $\partial B_{0}$. The existence of a solution $\left(E_{1}^{\ell}, \phi_{1}^{\ell}\right)$ of the linear eigenvalue problem

$$
\left.\begin{array}{l}
{\left[(-\Delta+V)+\lambda W\left(\psi_{0}\right)\right] \phi_{1}^{\ell}=E_{1}^{\ell} \phi_{1}^{\ell}} \\
\phi_{1}^{\ell} \in \ell^{2}\left(B_{1}\right), \quad\left\|\phi_{1}^{\ell}\right\|_{2}=1,\left.\quad \phi_{1}^{\ell}\right|_{\partial B_{1}}=0,
\end{array}\right\}
$$

satisfying the induction hypothesis $\mathscr{I}(1)$, can be proven easily, because $V+\lambda W\left(\psi_{0}\right)$ has a deep well near the origin which enables us to prove an analogue of Lemma 7.3 and of (7.7).

\section{Construction of $\boldsymbol{\Omega}_{n+1}$ and $B_{n+1}$}

Let $n$ be an integer $\geqq 1$, and suppose we know $\left(\Omega_{j}, B_{j}, E_{j}, \psi_{j}\right)$ for $j \leqq n$, and that the induction hypothesis $\mathscr{I}(j)$ holds for every $j \leqq n$. In this section we shall construct $\Omega_{n+1}$ and $B_{n+1}$ such that $\mathscr{I}_{1}(n+1), \mathscr{I}_{2}(n+1)$, and $\mathscr{I}_{3}(n+1)$ are fulfilled.

Let $A_{n+1}^{d} \equiv B_{n+1}^{d} \backslash B_{n}^{d}$, where $B_{n}^{d}=\left\{x:|x| \leqq d_{n}\right\}$. We define $\Omega_{n+1}$ to be the subset of $\Omega_{n}$ of potentials, $V$, satisfying the following conditions in the annulus $A_{n+1}^{d} \cup A_{n+2}^{d}$ :

$$
\operatorname{dist}\left(\sigma\left(H_{0}\left(A_{n+1}^{d}\right)\right), E_{n-1}\right) \geqq \delta_{n-1}
$$

and

$$
\left(A_{n+1}^{d} \cup A_{n+2}^{d}\right) \cap S_{n-1}\left(E_{n-1}, V\right)=\emptyset .
$$

The singular sets $S_{k}(E, V)$ appearing here are defined recursively. Namely, we set

$$
S_{0}(E, V)=\left\{x \in \mathbb{Z}^{v}:|V(x)-E| \leqq \frac{1}{2}|E|+2 v\right\}
$$

and if $0 \leqq k \leqq n-2$, we define

$$
S_{k+1}(E, V) \equiv S_{k}(E, V) \backslash S_{k}^{g}(E, V),
$$


where $S_{k}^{g}(E, V)=\bigcup_{\alpha} C_{k}^{\alpha}(E, V)$ is a maximal union of components satisfying

$$
\begin{gathered}
C_{k}^{\alpha}(E, V) \subset S_{k}(E, V) ; \\
\operatorname{diam} C_{k}^{\alpha}(E, V)<d_{k} ; \\
\operatorname{dist}\left(C_{k}^{\alpha}(E, V), S_{k}(E, V) \backslash C_{k}^{\alpha}(E, V)\right)>2 d_{k+1} ; \\
\operatorname{dist}\left(E, \sigma\left(H_{0}\left(\bar{C}_{k}^{\alpha}(E, V)\right)\right)\right)>2 \delta_{k},
\end{gathered}
$$

where

$$
\bar{C}_{k}^{\alpha}(E, V) \equiv\left\{x: \operatorname{dist}\left(x, C_{k}^{\alpha}(E, V)\right) \leqq 4 d_{k}\right\} .
$$

There are two probabilistic estimates we need in order to bound $\operatorname{Prob}\left(\Omega_{n+1} \backslash \Omega_{n}\right)$. The first one is a consequence of Wegner's results (see $[5,6]$ ).

Lemma 8.1. If $\Lambda$ is a $V$-independent subset of $\mathbb{Z}^{v}$ with cardinality $|\Lambda|<\infty$, then the probability of the event

$$
\operatorname{dist}\left(E, \sigma\left(H_{0}^{V}(\Lambda)\right)\right) \leqq \kappa
$$

is bounded from above by

$$
\min \left(c \kappa^{1 / 2} \varrho_{1}(E, \zeta)|\Lambda|, 2 \kappa \zeta^{-1}|\Lambda|\right),
$$

where $c$ is a constant and $\varrho_{1}(E, \zeta) \downarrow 0$, as $(\zeta+|E|) \uparrow \infty$.

On the basis of Theorem 2.2 in [6], we get also the following bound:

Lemma 8.2. There is a number $N$ such that if $|\widetilde{E}|+\zeta \geqq N$, then the probability that

$$
S_{k-1}\left(E_{k-1}, V\right) \cap \Lambda \neq \emptyset
$$

is less than $\varrho_{2}(\widetilde{E}, \zeta)|\Lambda| d_{k-1}^{-(2 v+2)}$, and $\varrho_{2}(\widetilde{E}, \zeta) \downarrow 0$, as $(\zeta+|\widetilde{E}|) \uparrow \infty$.

These two lemmas permit us to bound from above the probability that (8.1) and (8.2) fail to hold. Namely, we have

Prob $\{(8.1)$ or $(8.2)$ do not hold $\} \leqq \min \left(c_{v} \delta_{n-1}^{1 / 2} \varrho_{1}(\tilde{E}, \zeta)\left|A_{n+1}^{d}\right|, 2 \delta_{k-1} \zeta^{-1}\left|A_{n+1}^{d}\right|\right)$

$$
+\varrho_{2}(\tilde{E}, \zeta)\left|A_{n+1}^{d} \cup A_{n+2}^{d}\right| d_{n-1}^{-(2 v+2)} .
$$

For sufficiently large $d_{0}$, the second term dominates the first term. We have that

$$
\left|A_{n+1}^{d} \cup A_{n+2}^{d}\right| d_{n-1}^{-(2 v+2)} \leqq c_{v} d_{n+2}^{v} d_{n-1}^{-2(v+1)} \leqq c_{v} d_{n-1}^{-1},
$$

where $c_{v}$ is a geometric constant and $d_{n}=\left(d_{0}\right)^{(5 / 4)^{n}}$. This proves $\mathscr{I}_{1}(n)$.

Let us now pass to the construction of $B_{n+1}$. Let $\gamma_{n+1} \equiv \partial B_{n+1}$ and let $\gamma_{n+1}^{d} \equiv \partial B_{n+1}^{d}$, where the boundary of a set $A \subset \mathbb{Z}^{v}$ is defined as follows:

$$
\partial A=\left\{x, y \in \mathbb{Z}^{v}:|x-y|=1, x \in A \text { and } y \in \mathbb{Z}^{v} \backslash A \text { or vice versa }\right\} .
$$

Following [6], we require the following definition.

Definition. A set $A \subset \mathbb{Z}^{v}$ is $(k, E)$-admissible if

$$
\partial A \cap \overline{C_{j}^{\alpha}}=\emptyset, \quad \forall \alpha \quad \text { and } \quad \forall j=0,1, \ldots, k .
$$


From Appendix D of [6], it follows that there exists a $(n-1)$-admissible set satisfying $\mathscr{I}_{2}(n+1): B_{n+1}$ will be such a set.

Next we need the following lemma which establishes $\mathscr{I}_{3}(n+1)$.

Lemma 8.3. For $|\widetilde{E}|+\zeta$ large enough, for all $V \in \Omega_{k}$ and every $k=1, \ldots, n$, there exists a constant $m(\widetilde{E}, \zeta)>0$ such that

$$
\left|G_{A_{k}}^{\lambda, n}(x, y ; z)\right| \leqq \exp (-m(\tilde{E}, \zeta)|x-y|),
$$

provided

$$
\left|z-E_{n}\right| \leqq \frac{1}{2} \delta_{k-1} \quad \text { and } \quad|x-y| \geqq \frac{1}{5} d_{k+1} .
$$

Moreover, we have

$$
\operatorname{dist}\left(E_{n}, \sigma\left(H_{\lambda, n}^{V}\left(A_{k}\right)\right)\right) \geqq \frac{1}{2} \delta_{k-2}^{2}
$$

for every $k=2, \ldots, n$.

The proof of the decay estimate (8.17) is an almost immediate consequence of the proof of Theorem 2.1 in [6], while to verify (8.19) one needs some extra arguments. We defer the proof of this lemma to Appendix B.

\section{Construction of $\phi_{n+1}$}

We now assume that we know $\left(\Omega_{k}, B_{k}, \psi_{k}, E_{k}\right)$ for $k \leqq n$, and that these quadruples satisfy the first $n$ induction hypotheses. In Sect. 8 we constructed $\Omega_{n+1}$ and $B_{n+1}$ satisfying $\mathscr{I}_{1}(n+1), \mathscr{I}_{2}(n+1)$, and $\mathscr{I}_{3}(n+1)$. Here, we consider the following linear eigenvalue problem

$$
\left.\begin{array}{l}
\left(-\Delta+V+\lambda W\left(\psi_{n}\right)\right) \phi_{n+1}=E_{n+1}^{\ell} \phi_{n+1} \\
\phi_{n+1} \in \ell^{2}\left(B_{n+1}\right), \quad\left\|\phi_{n+1}\right\|_{2}=1,\left.\quad \phi_{n+1}\right|_{\partial B_{n+1}}=0 .
\end{array}\right\}
$$

Our aim is to prove that this eigenvalue problem has a solution $\left(E_{n+1}^{\ell}, \phi_{n+1}\right)$, which satisfies the first part of the induction hypotheses $\mathscr{I}_{4}(n+1), \mathscr{I}_{6}(n+1)$, and $\mathscr{I}_{7}(n+1)$.

Thanks to $\mathscr{I}_{4}(n), \mathscr{I}_{5}(n)$, and $\mathscr{I}_{6}(n)$, we have the following bound.

Lemma 9.1. We have

$$
\left\|G_{B_{n}}^{\lambda, n}(z)\right\| \leqq\left[\frac{1}{4} e^{-\sqrt{d_{n-1}}}-\|D W\| e^{-\frac{1}{2} m d_{n-1}}-e^{-\frac{1}{2} m d_{n-1}}\right]^{-1}
$$

for every $z \in \mathscr{C}_{n-1}=\left\{z:\left|z-E_{n-1}\right|=\delta_{n-1}\right\}$, where $D W$ and $\|D W\|$ are defined in Appendix D.

From the induction hypothesis $\mathscr{I}_{3}(n+1)$, verified in Sect. 8 , we also find the lemma

Lemma 9.2. We have

$$
\left\|G_{A_{k}}^{\lambda, n}(z)\right\| \leqq 2 \delta_{k-1}^{-1}, \quad \forall z \in \overline{\mathscr{C}}_{k-1, k \leqq n} .
$$

The existence of a solution of (9.1) with eigenvalue close to $E_{n}$, follows from the following lemma. 
Lemma 9.3. For $d_{0}$ large enough (independent of $n$ ), the Hamiltonian $H_{\lambda, n}^{V}\left(B_{n+1}\right)$ has one and only one eigenvalue in the interval

$$
\left[E_{n}-\frac{1}{2} \delta_{n}, E_{n}+\frac{1}{2} \delta_{n}\right]
$$

and we have

$$
\left\|G_{B_{n+1}}^{\lambda, n}(z)\right\| \| \leqq e^{2 \sqrt{d_{n}}} .
$$

In this and in the following section, we omit writing the superscript " $\lambda, n$ " on the Green functions $G_{B_{n}}^{\lambda, n}$ and $G_{B_{n+1}}^{\lambda, n}$.

Proof. It is enough to prove that $G_{B_{n+1}}(z)$ is analytic near $\mathscr{C}_{n}$ and that

$$
\left\|\frac{1}{2 \pi i} \oint_{\mathscr{C}_{n}}\left[G_{B_{n+1}}(z)-G_{B_{n}}(z)\right] d z\right\|<1 .
$$

In order to establish (9.6), we shall view the couplings across $B_{n}$ as a perturbation in which we propose to expand. To this end, we define coupling matrices $\Gamma_{n}$, corresponding to $\gamma_{n}=\partial B_{n}$, as follows:

$$
\Gamma_{n}(x, y)= \begin{cases}1 & \text { if }(x, y) \in \gamma_{n} \\ 0 & \text { otherwise }\end{cases}
$$

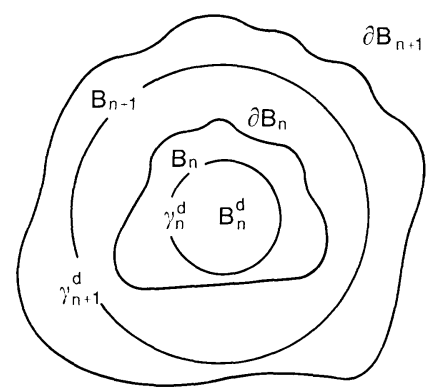

Fig. 2

Then

$$
H_{n-1}\left(B_{n+1}\right)=H_{n-1}\left(B_{n}\right) \oplus H\left(A_{n+1}\right)-\Gamma_{n} .
$$

Our bound on (9.6) follows from a resolvent identity. Let us define

$$
G_{n+1}(z)=G_{B_{n}}(z) \oplus G_{A_{n+1}}(z) .
$$

$G_{n+1}(z)$ is the resolvent of $H_{n-1}\left(B_{n}\right) \oplus H\left(A_{n+1}\right)$. From (9.9) we find

$$
G_{B_{n+1}}(z)=G_{n+1}(z)+\left[G_{n+1} \Gamma_{n} G_{B_{n+1}}\right](z) .
$$

Similarly, if we set

$$
G_{n+1}^{d}(z)=G_{B_{n+1} \backslash A_{n+1}^{d}}(z) \oplus G_{A_{n+1}^{d}+1}(z)
$$

(see Fig. 2 for the definition of $B_{n}, A_{n}, \ldots$ ), then we have

$$
G_{B_{n+1}}(z)=G_{n+1}^{d}(z)+G_{n+1}^{d}\left(\Gamma_{n}^{d}+\Gamma_{n+1}^{d}\right) G_{B_{n+1}}(z) .
$$


By alternate applications of (9.11) and (9.12) we find

$$
\begin{aligned}
G_{B_{n+1}}(z)= & G_{n+1}(z)+G_{n+1} \Gamma_{n} G_{n+1}^{d}(z)+G_{n+1} \Gamma_{n} G_{n+1}^{d}\left(\Gamma_{n}^{d}+\Gamma_{n+1}^{d}\right) G_{n+1}(z) \\
& +G_{n+1} \Gamma_{n} G_{n+1}^{d}\left(\Gamma_{n}^{d}+\Gamma_{n+1}^{d}\right) G_{n+1} \Gamma_{n} G_{B_{n+1}}(z) \\
\equiv & F(z)+L(z) G_{B_{n+1}}(z) .
\end{aligned}
$$

Hence

$$
G_{B_{n+1}}(z)-G_{B_{n}}(z)=F(z)-G_{B_{n}}(z)+L(z) G_{B_{n+1}}(z)
$$

where the definitions of $F$ and $L$ follow from (9.14).

By integrating the first term in (9.14), we obtain a result which cancels against the second term in (9.6). In fact,

$$
\begin{aligned}
\frac{1}{2 \pi i} \oint_{\mathscr{C}_{n}} G_{n+1}(z) d z & =\frac{1}{2 \pi i} \oint_{\mathscr{C}_{n}} G_{B_{n}}(z) d z+\frac{1}{2 \pi i} \oint_{\mathscr{C}_{n}} G_{A_{n+1}}(z) d z \\
& =\frac{1}{2 \pi i} \oint_{\mathscr{C}_{n}} G_{B_{n}}(z) d z=P_{\psi_{n}} .
\end{aligned}
$$

For the second term in (9.14), we have

$$
\begin{aligned}
\left\|\frac{1}{2 \pi i} \oint_{\mathscr{C}_{n}} G_{n+1} \Gamma_{n} G_{n+1}^{d} d z\right\| & =\left\|\frac{1}{2 \pi i} \oint_{\mathscr{C}_{n}} G_{B_{n}} \Gamma_{n} G_{A_{n+1}^{d}}\right\| \\
& =\left\|P_{\psi_{n}} \Gamma_{n} G_{A_{n+1}^{d}}\left(E_{n}\right)\right\| \leqq\left|\gamma_{n}\right| e^{-m d_{n}} \delta_{n}^{-1},
\end{aligned}
$$

where we have used the induction hypothesis on $\psi_{n}$. The integral of the third term can be bounded in a similar way,

$$
\begin{aligned}
& \left\|\frac{1}{2 \pi i} \oint_{\mathscr{C}_{n}}\left(G_{B_{n}} \oplus G_{A_{n+1}}\right) \Gamma_{n}\left(G_{B_{n+1} \backslash A_{n+1}^{d}} \oplus G_{A_{n+1}^{d}}\right)\left(\Gamma_{n}^{d}+\Gamma_{n+1}^{d}\right)\left(G_{B_{n}} \oplus G_{A_{n+1}}\right)\right\| \\
& =\| \frac{1}{2 \pi i} \oint_{\mathscr{C}_{n}}\left\{G_{B_{n}} \Gamma_{n} G_{A_{n+1}^{d}} \Gamma_{n}^{d} G_{B_{n}}+G_{B_{n}} \Gamma_{n} G_{A_{n+1}^{d}} \Gamma_{n+1}^{d} G_{A_{n+1}}\right. \\
& \left.\quad+G_{A_{n+1}} \Gamma_{n} G_{A_{n+1}^{d}} \Gamma_{n}^{d} G_{B_{n}}\right\} \| .
\end{aligned}
$$

To compute the integral in (9.17), let us introduce the pole subtracted Green function

$$
K_{B_{n}}(z)=G_{B_{n}}(z)-\frac{1}{z-E_{n}} P_{\psi_{n}},
$$

where $P_{\psi_{n}}$ is the orthogonal projection onto $\psi_{n}$. Equation (9.17) is equal to

$$
\begin{aligned}
& \| P_{\psi_{n}} \Gamma_{n} G_{A_{n+1}^{d}}\left(E_{n}\right) \Gamma_{n}^{d} K_{B_{n}}\left(E_{n}\right)+K_{B_{n}}\left(E_{n}\right) \Gamma_{n} G_{A_{n+1}^{d}}\left(E_{n}\right) \Gamma_{n}^{d} P_{\psi_{n}} \\
& \quad+P_{\psi_{n}} \Gamma_{n} G_{A_{n+1}^{d}}\left(E_{n}\right) \Gamma_{n+1}^{d} G_{A_{n+1}}\left(E_{n}\right)+G_{A_{n+1}}\left(E_{n}\right) \Gamma_{n} G_{A_{n+1}^{d}}\left(E_{n}\right) \Gamma_{n}^{d} P_{\psi_{n}} \| .
\end{aligned}
$$

Thanks to (8.1), (8.17), (6.17), (6.18), and (6.22), this expression can be bounded from above by

$$
96 v\left|\gamma_{n}\right| \exp \left(\sqrt{d_{n-1}}+\sqrt{d_{n+1}}-m d_{n}\right)
$$


To bound (9.15), what remains to be done is to bound the operator-norm of $L(z) G_{B_{n+1}}(z)$. We shall bound

$$
\left\|\Gamma_{n+1}^{d} G_{n+1}(z) \Gamma_{n}\right\|=\left\|\Gamma_{n+1}^{d} G_{A_{n+1}}(z) \Gamma_{n}\right\|
$$

and

$$
\left\|\Gamma_{n}^{d} G_{n+1}(z) \Gamma_{n}\right\|=\left\|\Gamma_{n}^{d} G_{B_{n}}(z) \Gamma_{n}\right\|
$$

separately, for $z \in \mathscr{C}_{n+1}$. From Lemma 8.6 we get

$$
\begin{aligned}
\left\|\Gamma_{n+1}^{d} G_{A_{n+1}} \Gamma_{n}\right\| & \leqq\left|\gamma_{n+1}^{d}\right|\left\|\Gamma_{n+1}^{d} G_{A_{n+1}} \Gamma_{n}\right\| \\
& \leqq\left|\gamma_{n+1}^{d}\right| \exp \left(-m\left(d_{n+1}-\frac{4}{5} d_{n}\right)\right) .
\end{aligned}
$$

To bound (9.21), let us observe that if $x \in \gamma_{n}^{d}$ and $y \in \gamma_{n}$ then, due to $\mathscr{I}_{2}(n)$, we have $|x-y| \geqq \frac{1}{2} d_{n}$. We can thus make use of Lemma 8.6 to bound the kernel of $G_{A_{n}}(z)$ for $z \in \mathscr{C}_{n}$, and we find

$$
\left|G_{A_{n}}(x, y ; z)\right| \leqq e^{-m|x-y|} \leqq e^{-\frac{m}{2} d_{n}}, \quad \forall(x, y) \in \gamma_{n}^{d} \times \gamma_{n} .
$$

Let us now recall the following resolvent identity

$$
\begin{aligned}
G_{B_{n}}(x, y ; z) & =\left(G_{n}+G_{n} \Gamma_{n-1} G_{n}+G_{n} \Gamma_{n-1} G_{B_{n}} \Gamma_{n-1} G_{n}\right)(x, y ; z) \\
& =\left(G_{A_{n}}+G_{A_{n}} \Gamma_{n-1} G_{B_{n}} \Gamma_{n-1} G_{A_{n}}\right)(x, y ; z)
\end{aligned}
$$

for all $x \in \gamma_{n}^{d}, y \in \gamma_{n}$. Hence, we have

$$
\begin{aligned}
\left\|\Gamma_{n}^{d} G_{B_{n}} \Gamma_{n}\right\| \leqq & \left|\gamma_{n}\right|\left|\gamma_{n}^{d}\right| e^{-\frac{1}{2} m d_{n}} \\
+ & {\left[\frac{1}{4} e^{-\sqrt{d_{n-1}}}-\|D W\| e^{-\frac{1}{2} m d_{n-1}}-e^{-\frac{1}{2} m d_{n-1}}\right]^{-1}\left|\gamma_{n-1}\right|^{2} } \\
& \times e^{-m\left(d_{n}-2 d_{n}-1\right)} e^{-m\left(d_{n}-\frac{3}{2} d_{n-1}\right)} .
\end{aligned}
$$

By collecting (9.16), (9.19), (9.20), and (9.23), we find that if $d_{0}$ is larger than a certain constant independent of $n$, then

$$
\left\|L(z) G_{B_{n+1}}(z)\right\| \leqq c_{1} e^{c_{2} \sqrt{d_{n+1}}-m d_{n}}\left|\gamma_{n}\right|\left|\gamma_{n}^{d}\right|
$$

and

$$
\begin{aligned}
& \left\|\frac{1}{2 \pi i} \oint_{\mathscr{C}_{n}}\left[G_{B_{n+1}}(z)-G_{B_{n}}(z)\right] d z\right\| \\
& \quad \leqq c\left|\gamma_{n}\right|\left|\gamma_{n}^{d}\right| e^{-(m / 2) d_{n}} .
\end{aligned}
$$

Moreover, if $z \in \mathscr{C}_{n}$, then using Lemma 9.1, (8.1) and $\mathscr{I}_{4}(n)$, we have

$$
\begin{aligned}
\left\|G_{B_{n+1}}(z)\right\| \leqq & \left|B_{n+1}\right|\left[\left\|G_{B_{n}}(z)\right\|+\left\|G_{A_{n+1}}(z)\right\|\right. \\
& \left.+2\left(\left|\gamma_{n}\right| e^{\sqrt{d_{n}}-m d_{n}}+8 v\left|\gamma_{n}\right|\left|\gamma_{n}^{d}\right| e^{2 \sqrt{d_{n}}-m d_{n}}\right)\right] \\
\leqq & \left|B_{n+1}\right|\left[\left(\frac{1}{4} e^{-\sqrt{d_{n}-1}}-\|D W\| e^{-\frac{1}{2} m d_{n-1}}-e^{-\frac{1}{2} m d_{n-1}}\right)^{-1}\right. \\
& \left.+\left(e^{-\sqrt{d_{n-1}}}-e^{-\frac{m}{2} d_{n-1}}\right)^{-1}+2\left(\left|\gamma_{n}\right| e^{\sqrt{d_{n}}-m d_{n}}+8 v\left|\gamma_{n}\right|\left|\gamma_{n}^{d}\right| e^{2 \sqrt{d_{n}}-m d_{n}}\right)\right] \\
\leqq & e^{2 \sqrt{d_{n}}}
\end{aligned}
$$

where the last step holds for $d_{0}$ large enough. Q.E.D. 
We can now pass to the proof of the induction hypothesis $\mathscr{I}_{4}(n+1)$, $\mathscr{I}_{6}(n+1)$, and $\mathscr{I}_{7}(n+1)$. Let us observe that $\phi_{n+1}$ can be represented as follows:

$$
\phi_{n+1}=\frac{c}{2 \pi i} \oint_{\mathscr{C}_{n}} G_{B_{n}+1}(z) \psi_{n} d z
$$

where $c$ is a positive normalization constant. Using again a resolvent identity, we find

$$
\begin{aligned}
\phi_{n+1} & =\frac{c}{2 \pi i} \oint_{\mathscr{E}_{n}}\left[G_{n+1}+G_{n+1} \Gamma_{n} G_{n+1}+G_{n+1} \Gamma_{n} G_{B_{n+1}} \Gamma_{n} G_{n+1}\right] \psi_{n} \\
& =c\left[\psi_{n}+G_{A_{n+1}}\left(E_{n}\right) \Gamma_{n} \psi_{n}+\frac{1}{2 \pi i} \oint_{\mathscr{G}_{n}} G_{n+1}(z) \Gamma_{n} G_{B_{n+1}}(z) \Gamma_{n} \psi_{n} \frac{d z}{\left(E_{n}-z\right)}\right] .
\end{aligned}
$$

The $\ell^{2}$-norms of the last two terms inside the square brackets are bounded above by

$$
\begin{aligned}
& \left\|G_{A_{n+1}}\left(E_{n}\right)\right\|\left\|\Gamma_{n} \psi_{n}\right\|_{\ell^{2}} \\
& \quad+\sup _{z \in \mathscr{C}_{n}}\left\{\left[\left\|G_{B_{n}}(z)\right\|+\left\|G_{A_{n+1}}(z)\right\|\right]\left\|G_{B_{n+1}}(z)\right\| \|_{\mathscr{L}\left(\ell^{2}\right)}\right\}\left\|\Gamma_{n} \psi_{n}\right\| \\
& \leqq 2\left|\gamma_{n}\right| e^{\sqrt{d_{n}-m d_{n}}+4 e^{\sqrt{d_{n}}} e^{2 \sqrt{d_{n}}}\left|\gamma_{n}\right| e^{-m d_{n}} \equiv a_{n} .}
\end{aligned}
$$

For the normalization factor $c$ we thus get the following estimates:

$$
1-a_{n} \leqq c^{-1} \leqq 1+a_{n},
$$

where $a_{n}$ is the constant defined in (9.29). Moreover, we have

$$
\begin{aligned}
& \left\|\phi_{n+1}-\psi_{n}\right\|_{\infty} \leqq(1-c)\left\|\psi_{n}\right\| \\
& \quad+c\left\|G_{A_{n+1}}\left(E_{n}\right) \Gamma_{n} \psi_{n}+\frac{1}{2 \pi i} \oint_{\mathscr{G}_{n}} G_{n+1}(z) \Gamma_{n} G_{B_{n+1}}(z) \Gamma_{n} \psi_{n} \frac{d z}{E_{n}-z}\right\| \\
& \leqq \\
& \quad(1-c)+c a_{n} \leqq\left(1-\frac{1}{1+a_{n}}\right)+\frac{a_{n}}{1-a_{n}}=\frac{2 a_{n}}{1-a_{n}^{2}} .
\end{aligned}
$$

Hence, if $d_{0}$ is larger than a certain constant depending on $\lambda$ but not on $n$, the bound (6.19) of the first part of $\mathscr{I}_{6}(n+1)$ is valid. The first part of $\mathscr{I}_{4}(n+1)$ derives from (9.29) and can be proven by writing $E_{n+1}^{\ell}$ and $E_{n}$ as the expectation value of the corresponding Hamiltonian in the corresponding eigenstate. Finally, the first part of $\mathscr{I}_{7}(n+1)$ derives from Lemma 8.6 once we represent $\left|\phi_{n+1}(x)\right|$ as follows:

$$
\left|\phi_{n+1}(x)\right|=\left|\sum_{|y|=1}\left(\tilde{H}-E_{n+1}^{\ell}\right)^{-1}(x, y)\left[\phi_{n+1}(y)-\phi(0)\right]\right| \leqq 2 v e^{-m|x|},
$$

where $\widetilde{H}=H_{n}\left(B_{n+1} \backslash\{\underline{0}\}\right)$ and the last step holds provided $|x| \geqq \frac{1}{5} d_{n+1}$.

\section{Construction of $\psi_{n+1}$. The Proof Completed}

As anticipated in Sect. 6 , in this section we shall construct a solution $\left(\psi_{n+1}, E_{n+1}\right)$ to the non-linear eigenvalue problem (6.2) in the ball $B_{n+1}$ by considering the 
following family of n.l.e.p.'s connecting (6.10) with (6.2):

$$
\left.\begin{array}{l}
\left(-\Delta+V+\lambda W\left(\psi_{n}\right)\right) u_{\beta}+\beta\left(W\left(u_{\beta}\right)-W\left(\psi_{n}\right)\right) u_{\beta}=E(\beta) u_{\beta} \\
u_{\beta} \in \ell^{2}\left(B_{n+1}\right), \quad\left\|u_{\beta}\right\|_{2}=1,\left.\quad u_{\beta}\right|_{\partial B_{n+1}}=0,
\end{array}\right\}
$$

where $\beta \in[0, \lambda]$, and we look for a solution $u_{\beta}(x)$ which depends smoothly on $\beta$ and satisfies the condition

$$
\left.u_{\beta}(x)\right|_{\beta=0}=\phi_{n+1}(x)
$$

If $\beta$ is so small that

$$
\left\|u_{\beta}-\psi_{n}\right\|_{\infty}<\frac{\delta_{n}}{2 \beta\|D W\|}
$$

we have

$$
\left\|\beta\left(W\left(u_{\beta}\right)-W\left(\psi_{n}\right)\right)\right\|_{\infty}<\frac{1}{2} \delta_{n},
$$

and Eq. (10.1) can be rewritten in integral form

$$
u_{\beta}(x)=\frac{c\left(\beta, u_{\beta}\right)}{2 \pi i} \oint_{\mathscr{C}_{n}}\left(z+\Delta-V-\lambda W\left(\psi_{n}\right)-\beta\left[W\left(u_{\beta}\right)-W\left(\psi_{n}\right)\right]\right)^{-1} \phi_{n+1},
$$

where $c\left(\beta, u_{\beta}\right)$ is a positive normalization constant. Recalling the definition (6.6) and (6.7) of the Green functions and the convention stated in Sect. 9 to omit the superscript $n$, we can expand (10.5) in a geometric series as follows:

$$
u_{\beta}(x)=c\left[\phi_{n+1}+\sum_{j=1}^{\infty} \beta^{j} \frac{1}{2 \pi i} \oint_{\mathscr{C}_{n}} G_{B_{n+1}}\left[\left(W\left(u_{\beta}\right)-W\left(\psi_{n}\right)\right) G_{B_{n+1}}\right]^{j} \phi_{n+1}\right] \text {. }
$$

Due to the implicit function theorem, [8], we know that the problems (10.1) and (10.6) have a solution for $\beta$ small. In this case, the solution $u_{\beta}$ obeys the differential equation one gets by differentiating both sides of (10.6) with respect to $\beta$ :

$$
\begin{aligned}
\frac{d u_{\beta}}{d \beta}(x)= & c\left(\beta, u_{\beta}\right)\left(1-P_{u_{\beta}}\right)\left\{\sum_{j=1}^{\infty} j \beta^{j-1} \frac{1}{2 \pi i} \oint_{\mathscr{C}_{n}} G_{B_{n+1}}\left[\left(W\left(u_{\beta}\right)-W\left(\psi_{n}\right)\right) G_{B_{n+1}}\right]^{j} \phi_{n+1}\right. \\
& \left.+\sum_{j=1}^{\infty} \beta^{j} \frac{1}{2 \pi i} \oint_{\mathscr{C}_{n}} G_{B_{n+1}} \frac{d}{d \beta}\left[\left(W\left(u_{\beta}\right)-W\left(\psi_{n}\right)\right) G_{B_{n+1}}\right]^{j} \phi_{n+1}\right\}(x) \\
\equiv & c(\beta, u)\left(F_{1}\left(\beta, u_{\beta}\right)(x)+\sum_{y} F_{\beta}\left(\beta, u_{\beta}\right)(x, y) \frac{d u_{\beta}}{d \beta}(y)\right) \\
= & c\left(F_{1}+F_{2}\left[\frac{d u_{\beta}}{d \beta}\right]\right)
\end{aligned}
$$

where $F_{1}$ and $F_{2}$ are defined in the obvious manner. Let $\left[0, \beta_{1}\right)$ be the maximal interval for which a solution of (10.1) exists, (10.3) holds and

$$
c\left\|F_{2}[\cdot]\right\| \mid<1 .
$$

Then, for all $\beta \in\left[0, \beta_{1}\right)$ we have

$$
\frac{d u_{\beta}}{d \beta}=\left(1-c F_{2}[\cdot]\right)^{-1} F_{1} .
$$


Our aim is to verify a priori bounds on the right-hand side of (10.9) which guarantee that $\beta_{1}>\lambda$ and that $\psi_{n+1} \equiv u_{\lambda}$ fulfills the $(n+1)^{\text {st }}$ induction hypothesis.

Let us introduce the following notation:

$$
\mathscr{U}_{n+1}=\left\{u \mid\left\|u-\psi_{n}\right\|_{\infty} \leqq \frac{1}{2} \min \left(1, \lambda^{-1}\right) e^{-\frac{1}{2} m d_{n}}\right\} .
$$

The estimates we need are contained in the following lemma:

Lemma 10.1. For $u_{\beta} \in \mathscr{U}_{n+1}$ and $\beta \leqq \lambda$, we have

$$
\begin{gathered}
c(\beta, u)=1+c_{0}\left(d_{0}, n\right), \\
\left\|F_{1}\left(u_{\beta}, \beta\right)\right\|_{\infty} \leqq c_{1}\left(d_{0}, U, n\right)\left\|u_{\beta}-\psi_{n}\right\|_{\infty}, \\
\left\|F_{2}\left(u_{\beta}, \beta\right)\right\| \leqq c_{2}\left(d_{0}, U, n\right),
\end{gathered}
$$

where $c_{0}, c_{1}$, and $c_{2}$ are constants $\downarrow 0$ uniformly in $n$, as both $d_{0}$ and $U \uparrow \infty$.

Before proving this lemma, let us show how it can be used to control the solutions of (10.9). We have

$$
\begin{aligned}
\frac{d}{d \beta}\left\|u_{\beta}-\phi_{n+1}\right\|_{\infty} & \leqq\left\|\frac{d u_{\beta}}{d \beta}\right\|_{\infty}=\left\|c\left(1-c F_{2}\right)^{-1} F_{1}\right\|_{\infty} \\
& \leqq\left(1+c_{0}\right)\left[1-\left(1+c_{0}\right) c_{2}\right]^{-1} c_{1}\left\|u_{\beta}-\psi_{n}\right\|_{\infty} \\
& \equiv c_{3}\left\|u_{\beta}-\psi_{n}\right\|_{\infty},
\end{aligned}
$$

where $c_{3} \downarrow 0$ uniformly in $n$, as $\left(U, d_{0}\right) \uparrow(\infty, \infty)$. We thus find the differential inequality

$$
\frac{d}{d \beta}\left\|u_{\beta}-\phi_{n+1}\right\|_{\infty} \leqq c_{3}\left(\left\|u_{\beta}-\phi_{n+1}\right\|_{\infty}+\left\|\psi_{n}-\phi_{n+1}\right\|_{\infty}\right)
$$

which, upon integration, yields the bound

$$
\left\|u_{\beta}-\phi_{n+1}\right\|_{\infty} \leqq\left(e^{\beta c_{3}}-1\right)\left\|\psi_{n}-\phi_{n+1}\right\|_{\infty} \leqq\left(e^{\beta c_{3}}-1\right) e^{-\frac{1}{2} m d_{n}} .
$$

From here it is clear that, if the depth, $U$, of the well at 0 and $d_{0}$ are larger than certain constants independent of $n$, then $u_{\beta} \in \mathscr{U}_{n+1}, \forall \beta \in[0, \lambda]$. Moreover, if we set $\psi_{n+1} \equiv u_{\lambda}$, the induction hypotheses $\mathscr{I}_{4}(n+1)$ and $\mathscr{I}_{6}(n+1)$ hold. The second part of $\mathscr{I}_{7}(n+1)$ can then be verified as the first part and this concludes the proof of $\mathscr{I}(n+1)$.

Proof of Lemma 10.1. (i) We have

$$
\begin{aligned}
c\left(\beta, u_{\beta}\right) & =\left|\left(\phi_{n+1}, u_{\beta}\right)\right|^{-1} \leqq|1-|\left|u_{\beta}-\phi_{n+1} \|_{2}\right|^{-1} \\
& \leqq|1-| B_{n+1}\left|e^{-\frac{m}{2} d_{n}}\right|^{-1} \equiv 1+c_{0}\left(d_{0}, n\right),
\end{aligned}
$$

where $c_{0}\left(d_{0}, n\right) \downarrow 0$ uniformly in $n$, as $d_{0} \uparrow \infty$.

(ii) For all $\beta \in[0, \lambda]$, we have

$$
\begin{aligned}
& \left\|F_{1}\left(u_{\beta}, \beta\right)\right\|_{\infty} \leqq\left\|K_{B_{n+1}}\left(E_{n+1}^{\ell}\right)\left(W\left(u_{\beta}\right)-W\left(\psi_{n}\right)\right) \phi_{n+1}\right\|_{\infty} \\
& \cdot+\sum_{j=2}^{\infty} j \lambda^{j-1}\left\|\frac{1}{2 \pi i} \oint_{\mathscr{C}_{n}} G_{B_{n+1}}(z)\left[\left(W\left(u_{\beta}\right)-W\left(\psi_{n}\right)\right) G_{B_{n+1}}(z)\right]^{j} \phi_{n+1}\right\|_{\infty},
\end{aligned}
$$


where $F_{1}$ and $K$ have been defined in (10.7) and (9.18), respectively. We shall bound the first and the second term of (10.16) separately. For the first one, we have:

$$
\begin{aligned}
& \left\|K_{B_{n+1}}\left(E_{n+1}^{\ell}\right)\left(W\left(u_{\beta}\right)-W\left(\psi_{n}\right)\right) \phi_{n+1}\right\|_{\infty} \leqq\|D W\|\left\|u_{\beta}-\psi_{n}\right\|_{\infty} \\
& \quad \times \sup _{x \in B_{n+1}}\left\{\sum_{y \in B_{n+1}}\left|K_{B_{n+1}}\left(E_{n+1}^{\ell}\right)(x, y) \| \phi_{n+1}(y)\right|\right\} .
\end{aligned}
$$

The supremum on the right-hand side is bounded above by

$$
\begin{aligned}
& \sup _{\substack{x \in B_{n+1} \\
|y| \leqq d_{0}}}\left|K_{B_{n+1}}\left(E_{n+1}^{\ell}\right)(x, y)\right| \sum_{|y| \leqq d_{0}}\left[e^{-m_{0}(U)|y|}+\sum_{j=0}^{n} e^{-\frac{m}{2} d_{n}}\right] \\
& \quad+\sum_{k=1}^{n+1} \sum_{d_{k-1}<|y|<d_{k}} \sup _{x \in B_{n+1}}\left|K_{B_{n+1}}\left(E_{n+1}^{\ell}\right)(x, y)\right|\left[e^{-m|y|}+\sum_{j=k}^{n} e^{-\frac{m}{2} d_{j}}\right] .
\end{aligned}
$$

In order to bound (10.18) we need a lemma which is quite similar to Lemma 3.1 in $[1]$.

Lemma 10.2. If $d_{0}$ is large enough, we have

$$
\sup _{x \in B_{n+1}}\left|K\left(E_{n+1}^{\ell}\right)(x, y)\right| \leqq \begin{cases}c_{4}(U)+c_{5}\left(d_{0}\right), & \forall 0 \leqq|y| \leqq d_{0} \\ 2 e^{\sqrt{d_{k}+1}}+c_{6}\left(d_{0}\right), & \forall d_{k} \leqq|y| \leqq d_{k+1}\end{cases}
$$

where $c_{4}(U) \downarrow 0$, as $U \uparrow \infty$, and $c_{5}\left(d_{0}\right), c_{6}\left(d_{0}\right) \downarrow 0$, as $d_{0} \uparrow \infty$.

Postponing the proof of this lemma to the end of this section, let us now use it to bound (10.18). We have

$$
\begin{aligned}
(10.18) \leqq & \left(c_{4}(U)+c_{5}\left(d_{0}\right)\right)\left(\sum_{y} e^{-m_{0}(U)|y|}+\left|B_{0}\right| \sum_{j=0}^{\infty} e^{-\frac{m}{2} d_{j}}\right) \\
& +\sum_{k=0}^{n}\left(2 e^{\sqrt{d_{k+1}}}+c_{6}\left(d_{0}\right)\right)\left(e^{-m d_{k-1}}+\left|A_{k+1}\right| \sum_{j=k}^{\infty} e^{-\frac{m}{2} d_{\jmath}}\right) \\
\equiv & c_{7}\left(U, d_{0}\right),
\end{aligned}
$$

where $c_{7}\left(U, d_{0}\right) \downarrow 0$ as $\left(U, d_{0}\right) \uparrow(\infty, \infty)$ and does not depend on the iteration step $n$.

Lemma 10.2 allows us to bound the series appearing in (10.16). In fact, we have

$$
\begin{aligned}
\sum_{j=2 .}^{\infty}(\cdot) & \leqq \sum_{j=2}^{\infty} j \lambda^{j-1}\left\|\frac{1}{2 \pi i} \oint_{\mathscr{C}_{n}} G_{B_{n+1}}\left[\left(W\left(u_{\beta}\right)-W\left(\psi_{n}\right)\right) G_{B_{n+1}}\right]^{j} \phi_{n+1}\right\|_{2} \\
& \leqq \sum_{j=2}^{\infty} j \lambda^{j-1} \sup _{z \in \mathscr{C}_{n}}\left\|G_{B_{n+1}}(z)\right\|^{j}\|D W\|^{j}\left\|u_{\beta}-\psi_{n}\right\|_{\infty}^{j} \\
& \leqq \sum_{j=2}^{\infty} j \lambda^{j-1}\|D W\|^{j} \exp \left(2 j \sqrt{d_{n}}-\frac{1}{2} j m d_{n}\right) \\
& =\|D W\| \exp \left(2 \sqrt{d_{n}}-\frac{1}{2} m d_{n}\right)\left[\left(1-\lambda\|D W\| \exp \left(2 \sqrt{d_{n}}-\frac{1}{2} m d_{n}\right)\right)^{-2}-1\right] \\
& \leqq\|D W\| \exp \left(2 \sqrt{d_{0}}-\frac{m}{2} d_{0}\right)\left[\left(1-\lambda\|D W\| \exp \left(2 \sqrt{d_{0}}-\frac{1}{2} m d_{0}\right)\right)^{-2}-1\right] \\
& \equiv c_{8}\left(d_{0}\right),
\end{aligned}
$$

where $c_{8}\left(d_{0}\right) \downarrow 0$ as $d_{0} \uparrow \infty$. 
By summing (10.20) and (10.21) we thus get (10.12).

(iii) To prove (10.13), we can begin with a bound similar to (10.16)

$$
\begin{aligned}
\left\|F_{2}\right\|_{\mathscr{L}(\ell \infty)}= & \sup _{\|v\|_{\infty}=1}\left\|F_{2} v\right\|_{\infty} \\
\leqq & \sup _{\|v\|_{\infty}=1}\left\{|\lambda|\left\|K_{B_{n+1}}\left(E_{n+1}^{\ell}\right) D W[v] \phi_{n+1}\right\|_{\infty}\right. \\
& +\sum_{j=2}^{\infty} \lambda^{j} \| \frac{1}{2 \pi i} \oint_{\mathscr{C}_{n}} \sum_{y \in B_{n+1}} v(y) \frac{\partial}{\partial u_{\beta}(y)} G_{B_{n+1}} \\
& \left.\times\left[\left(W\left(u_{\beta}\right)-W\left(\psi_{n}\right)\right) G_{B_{n+1}}\right]^{j} \phi_{n+1} \|_{\infty}\right\} .
\end{aligned}
$$

The structure of the two terms of (10.22) is very similar to that of the two terms of (10.16) and they can be treated in a similar way. For this reason, we shall omit the details of these bounds.

Proof of Lemma 10.2. The method used to prove Lemma 3.1 can be applied also to the present case and gives the following bound:

$$
\left[K_{B_{n+1}}\left(x, y ; E_{n+1}^{\ell}\right) \mid \leqq\left\{\begin{array}{lc}
\sup _{\substack{x, y \in B_{0} \\
\left|z-E_{n+1}^{\ell}\right|=\frac{1}{2} \gamma_{0}}}\left|G_{B_{1}}(x, y ; z)\right|+c_{5}\left(d_{0}\right) & \text { for } x, y \in B_{0} \\
2 e^{\sqrt{d_{k}}+c_{6}\left(d_{0}\right)} & \text { for }|x-y| \leqq \frac{1}{5} d_{k}, \\
e^{-\bar{m}(\tilde{E})|x-y|} & |y| \leqq d_{k}
\end{array}\right.\right.
$$

where $\gamma_{0}=\operatorname{dist}\left(E_{0}, \sigma\left(H_{0}\left(B_{0}\right)\right) \backslash\left\{E_{0}\right\}\right), \quad c_{5}\left(d_{0}\right)$ and $c_{6}\left(d_{0}\right) \downarrow 0$ as $d_{0} \uparrow \infty$, and $\bar{m}(\widetilde{E})$ $>\frac{1}{4} m\left(E_{0}\right)>0$ for $d_{0}$ large enough. Moreover, due to Lemma 7.2, we have that the supremum on the upper line of (10.23) $\downarrow 0$ faster than const $U^{-1}$ as $U \uparrow \infty$. Thus (10.19) follows from (10.23). Q.E.D.

\section{Appendix A. Replacing "Large $U$ " by a "Large Gap"}

To prove Theorem NL2, we have shown how to construct an infinite number of solutions of the n.l.e.p. (5.1) whose eigenvalues form a dense subset of $\left\{E:|E|>E_{1}^{\prime}(v, \zeta)\right\}$. Here, we wish to point out that our methods allow us to construct further solutions.

Let us suppose that the potential $V$ is chosen such that the problem

$$
\left.\begin{array}{l}
-\Delta \psi_{1}+V \psi_{1}+\lambda W\left(\psi_{1}\right) \psi_{1}=E_{1} \psi_{1} \\
\psi_{1} \in \ell^{2}\left(B_{1}^{d}\right),\left.\quad \psi_{1}\right|_{\partial B_{1}^{d}}=0, \quad\left\|\psi_{1}\right\|_{2}=1,
\end{array}\right\}
$$

admits a solution $\left(\psi_{1}, E_{1}\right)$, and that $E_{1}$ is a simple eigenvalue of the linear operator $-\Delta+V+\lambda W\left(\psi_{1}\right)$ separated by a large gap $\gamma_{0}$ from the rest of the spectrum. This is the property established in Lemma 7.2. It plays a basic role in the estimates of Sect. 10. For every fixed $\lambda$, such potentials, $V$, exist and form a subset of positive measure. In fact, notice that there is a constant such that $\sup _{\|\psi\|=1}\|\lambda W(\psi)\|_{\mathscr{L}\left(\ell^{2}\left(B_{1}\right)\right)}$ $=$ const $|\lambda|$, for all $\lambda$. If $V$ is a potential such that the problem (A.1) with $\lambda=0$ has an 
eigenvalue $E$ separated by a gap $\geqq \gamma_{0}+$ const $|\lambda|$ from the rest of the spectrum, then one can easily prove that a solution $\left(\psi_{1}, E_{1}\right)$ with the properties specified above, exists. The large gap permits us to control the exponential decay of $\psi_{1}$. In fact, if $x_{0}$ is the point where $\psi_{1}(x)$ takes its maximum, we have

$$
\psi_{1}(x)=\frac{1}{2 \pi i\left|\psi\left(x_{0}\right)\right|} \oint_{\mathscr{C}} d z \frac{1}{z+\Delta-V-\lambda W\left(\psi_{1}\right)} \delta\left(\cdot-x_{0}\right),
$$

and the Green function appearing here can be controlled with the aid of a CombesThomas argument; see Appendix C. If we adjust things in such a way that $x_{0}$ is far away from the boundary of $B_{1}^{d}$, then the solution $\psi_{1}(x)$ can be used as the starting point in our construction.

\section{Appendix B. Proof of Lemma 8.3}

The annulus $A_{k}$ is a $(k-3)$-admissible set such that

$$
A_{k} \cap S_{k-2}\left(E_{k-2}, V\right)=\emptyset
$$

and

$$
\sup _{x \in A_{k}}\left|W\left(\psi_{n}\right)(x)\right| \leqq 2 v e^{-m d_{k-1}}
$$

Since

$$
\left|E_{n}-E_{k-2}\right| \leqq \sum_{j=k-2}^{n} e^{-\frac{m}{2} d_{j}} \ll \frac{1}{4} \delta_{k-2}^{2},
$$

to prove (8.19) it will suffice to verify that

$$
\operatorname{dist}\left(\sigma\left(H_{\lambda, n}^{V}\left(A_{k}\right)\right), E_{k-2}\right) \geqq \frac{3}{4} \delta_{k-2}^{2},
$$

as long as $d_{0}$ is chosen large enough. During this proof we shall also argue that the exponential decay estimate (8.17) is true.

Let us introduce the modified family of singular sets, $\widetilde{S}_{j}\left(E_{k-2}, V\right)$, which are defined in the same way as the singular sets $S_{j}\left(E_{k-2}, V\right)$ were defined in Sect. 8, i.e. they are the union of components $\bigcup_{\alpha, r \geqq j} \widetilde{C}_{r}^{\alpha}\left(E_{k-2}, V\right)$, except that the condition (8.8) is replaced by the following one:

$$
\operatorname{dist}\left(E_{k-2}, \sigma\left(\left(H_{0}^{V}+\lambda W\left(\psi_{n}\right)\right)\left(\overline{\widetilde{C}}_{k-3}^{\alpha}\left(E_{k-2}, V\right)\right)\right)\right) \geqq \delta_{k-2} .
$$

This condition is strictly weaker than (8.8), in the sense that from (B.1) and (B.2) it follows that

$$
A_{k} \cap \widetilde{S}_{k-2}\left(E_{k-2}, V\right)=\emptyset .
$$

Moreover, $A_{k}$ is $(k-3)^{2}$-admissible in the following sense:

$$
\partial A_{k} \cap \widetilde{\widetilde{C}}_{j}^{\alpha}=\emptyset, \quad \forall j=0, \ldots, k-3 .
$$

These conditions are sufficient to apply the perturbation theory developed in [6] to prove Theorem 2.2. In this way we can arrive at the exponential decay estimate (8.17). 
One can also prove (B.4) following the lines of the proof of Theorem 2.2 of [6], though, in this case, some changes are required. If we denote by $\left(\mathscr{I}_{k}\right)$ the following induction hypothesis:

$\left(\mathscr{I}_{k}\right)$ : If $A \subset \mathbb{Z}^{v}$ is a set sharing with $A_{k}$ the properties (B.6) and (B.7), then (B.4) holds, or, because of $(8.17)$

$$
\left|G_{A}^{\lambda, n}\left(x, y ; E_{k-2}+i \varepsilon\right)\right| \leqq 4 \delta_{k-2}^{-1}
$$

for all $\varepsilon$ real $\neq 0$ and all $x, y \in A$.

Then one can prove that $\left(\mathscr{I}_{k-1}\right) \rightarrow\left(\mathscr{I}_{k}\right)$, for all $k \geqq 3$. Since $\left(\mathscr{I}_{2}\right)$ is evident, this implies (B.4). We underline that although the techniques in [6] are sufficient to prove (B.8), this result does not follow from Theorem 2.2 in [6]. In fact, this is a decay estimate on the Green function which holds only when the arguments $x$ and $y$ are enough separated, while we do not have such a restriction here.

To prove that $\left(\mathscr{I}_{k-1}\right) \rightarrow\left(\mathscr{I}_{k}\right)$, let us consider a subset $R^{\alpha}$ of $A$ which satisfies the $(k-3)^{\tilde{y}}$-admissibility condition (B.7), contains one and only one component $\widetilde{C}_{k-3}^{\alpha}$ of $\widetilde{S}_{k-3}^{g}$ and is such that

$$
\frac{1}{5} d_{k-2} \leqq \operatorname{diam} R^{\alpha} \leqq \frac{3}{2} d_{k-2}
$$

and

$$
\operatorname{dist}\left(\partial R^{\alpha}, \overline{\widetilde{C}_{k-3}^{\alpha}}\right)>0
$$

The existence of regions $R^{\alpha}$ with the properties above is checked in Appendix D of [6]. We have

Lemma B.1. Let $R \equiv R^{\alpha}$ be as above, and choose $d_{0}$ and $|\widetilde{E}|+\zeta$ sufficiently large, independently of $k$. Then

$$
\left|G_{R}\left(x, y ; E_{k-2}+i \varepsilon\right)\right| \leqq 2 \delta_{k-2}^{-1}
$$

for all $\varepsilon$ real $\neq 0$.

Proof. Let us introduce the simplified notations $E=E_{k-2}, C=\widetilde{C}_{k-3}^{\alpha}, \bar{C}=\overline{\widetilde{C}_{k-3}^{\alpha}}$. Let $B$ be a $(k-4)^{2}$-admissible set such that

$$
\left.\begin{array}{l}
\bar{C} \supset B \supset C \\
\operatorname{dist}(\sim B, C) \geqq 3 d_{k-3} \\
\operatorname{dist}(B, \sim \bar{C}) \geqq d_{k-3}
\end{array}\right\}
$$

and let $\bar{\gamma}=\partial \bar{C}, \gamma=\partial B, Q=R \backslash B, \bar{Q}=R \backslash \bar{C}$. We shall distinguish three cases

(i) $x \in B, y \in B$;

(ii) $x \in Q, y \in Q$;

(iii) $x \in B, y \in Q$.

In case (i), our lemma is a consequence of Sublemma 2.3 of [6]. In case (ii), we can use the following resolvent identity:

$$
G_{R}=G_{Q} \oplus G_{B}+\left(G_{Q} \oplus G_{B}\right) \Gamma\left(G_{\bar{Q}} \oplus G_{\bar{C}}\right)+\left(G_{Q} \oplus G_{B}\right) \Gamma G_{R} \bar{\Gamma}\left(G_{\bar{Q}} \oplus G_{\bar{C}}\right),
$$


where $\Gamma$ and $\bar{\Gamma}$ are the boundary operators related to $\gamma$ and $\bar{\gamma}$, respectively. If $x \in Q$ and $y \in Q$, we have

$$
\begin{aligned}
G_{R}(x, y ; E+i \varepsilon)= & G_{Q}(x, y ; E+i \varepsilon)+G_{Q} \Gamma G_{\bar{C}}(x, y ; E+i \varepsilon) \\
& +G_{Q} \Gamma G_{\bar{Q}}(x, y ; E+i \varepsilon)+G_{Q} \Gamma G_{R} \bar{\Gamma} G_{\bar{Q}}(x, y ; E+i \varepsilon) .
\end{aligned}
$$

Due to the induction hypothesis $\left(\mathscr{I}_{k-1}\right)$, we have

$$
\left|G_{Q}(x, y ; E+i \varepsilon)\right| \leqq 4 \delta_{k-3}^{-1},
$$

and the same inequality holds with $Q$ replaced by $\bar{Q}$. Thanks to (B.5), we have

$$
\left|G_{\bar{C}}(x, y ; E+i \varepsilon)\right| \leqq \delta_{k-2}^{-1} .
$$

Finally, by virtue of (B.12) and Theorem 2.2 of [6], we find

$$
\left|\Gamma G_{R} \bar{\Gamma}(x, y ; E+i \varepsilon)\right| \leqq|\gamma||\bar{\gamma}| e^{-m d_{k-3}} .
$$

These inequalities permit us to derive (B.11) from (B.15).

In case (iii), $x \in B$ and $y \in Q$, we can use the resolvent identity

from which we find

$$
G_{R}=G_{Q} \oplus G_{B}+G_{R} \Gamma\left(G_{Q} \oplus G_{B}\right)
$$

$$
G_{R}(x, y ; E+i \varepsilon)=G_{R} \Gamma G_{Q}(x, y ; E+i \varepsilon) .
$$

Thanks to Sublemma 2.3 of [6], we have

$$
\left|G_{R}(u, v ; E+i \varepsilon)\right| \leqq 2 \delta_{k-2}^{-1}
$$

for all $u, v \in B$. Hence, the bound (B.11) derives from (B.20) and Theorem 2.2 of [6]. Q.E.D.

To complete the proof of Lemma 8.3 one can make use of a method first used in [7], as in [6]. This method goes as follows: For every point $p \in A$ there exists a $(k-3)$-admissible set $R_{p}$ such that

$$
\operatorname{dist}\left(p, \partial R_{p} \backslash \partial A\right) \geqq \frac{1}{2} d_{k-2},
$$

and (B.9) holds. This is proven in Appendix D of [6]. One starts with $p=x$ and the resolvent identity

$$
G_{A}=G_{R_{x}} \oplus G_{A \sim R_{x}}+\left(G_{R_{x}} \oplus G_{A \backslash R_{x}}\right) \Gamma_{\partial R_{x}} G_{A} .
$$

In the second term, there are only matrix elements $G_{A}(u, y ; E+i \varepsilon)$ with $u \in \partial R_{x}$. For each $u \in \partial R_{x}$ one can then apply another resolvent identity similar to (B.22), but with $x$ replaced by $u$. Iterating this process, we find a series that converges rapidly thanks to the exponential decay estimates of [6]. The remainder of this series can be controlled in the same way as in [6], and using Lemma B.1, instead of Lemma 3.1 in [6], we can easily complete the proof of Lemma 8.3.

\section{Appendix C}

Let $\widetilde{H}=H_{\lambda, 0}\left(B_{0} \backslash\{\underline{0}\}\right)$. We have

$$
\left(\left(\widetilde{H}-E_{0}\right) \psi_{0}\right)(x)=-\psi_{0}(\underline{0}) \sum_{|y|=1} \delta(x-y),
$$


and so we find

$$
\left|\psi_{0}(x)\right|=\left|-\psi_{0}(\underline{0}) \sum_{|y|=1}\left(\tilde{H}-E_{0}\right)^{-1}(x, y)\right| \leqq 2 v \sup _{|y|=1}\left|\left(\tilde{H}-E_{0}\right)^{-1}(x, y)\right| .
$$

To prove Lemma 7.4 it is sufficient to prove that

$$
\left|\left(\tilde{H}-E_{0}\right)^{-1}(x, y)\right| \leqq \varepsilon^{-1} \exp (-m(\gamma, \varepsilon)|x-y|),
$$

where $m(\gamma, \varepsilon)=\ln \left(1+\frac{1}{2 v}(\gamma-\varepsilon-2 v)\right)$. To this end, let us introduce the operator

$$
\tilde{H}^{a} \equiv e^{-i a \cdot x} \tilde{H} e^{i a \cdot x}=\tilde{H}-\left[\sum_{|y|=1}\left(e^{i a \cdot y}-1\right) T_{y}\right],
$$

where $T_{y}$ is the operator such that $\left(T_{y} \psi\right)(x)=\psi(x+y)$. The norm of the operator within the square brackets in (C.3) is bounded above by

$$
\sum_{|y|=1}\left|e^{i a \cdot y}-1\right| \leqq 2 v\left[e^{|a|}-1\right]
$$

Hence, if $a=\operatorname{im}(\gamma, \varepsilon)$ the right-hand side of (C.4) is $(\gamma-\varepsilon-2 v)$, and we have

$$
\left|e^{i a \cdot(x-y)}\left(\tilde{H}-E_{0}\right)^{-1}(x, y)\right|=\left|\left(\tilde{H}^{a}-E_{0}\right)^{-1}(x, y)\right| \leqq \varepsilon^{-1},
$$

from which (C.2) follows.

\section{Appendix D}

Here we collect some of the most important notations we used. If $A \subset \mathbb{Z}^{v}$ is a subset and $\Delta(A)$ is the Laplacian on $\ell^{2}(A)$ with Dirichlet boundary conditions on $\partial A$, we set

$$
G_{A}^{\lambda, n}(z)=\left(z-\Delta(A)-V-\lambda W\left(\psi_{n}\right)\right)^{-1} .
$$

The superscripts " $\lambda, n$ " are omitted in Sect. 8 and Sect. 9. The operator $W\left(\psi_{n}\right)$ is defined in (5.4) where $W(|x-y|)$ is a function satisfying the decay estimate (5.5). If $u, v \in \ell^{2}\left(\mathbb{Z}^{v}\right)$, we denote by $D W[v]$ the operator on $\ell^{2}\left(\mathbb{Z}^{v}\right)$ such that

$$
D W[v] u \equiv 2 \sum_{x, y} W(|x-y|) u(x) v(y) .
$$

We denote by $\|D W\|$ the number

$$
\sup _{\|u\|_{2}=\|v\|_{2}=1}\|D W[v] u\|_{\infty} .
$$

When the symbol $\|\cdot\|$ contains a linear operator on $\ell^{2}(A), A \subset \mathbb{Z}^{v}$, it denotes the operator norm. Moreover \|\|$\cdot \|$ denotes the operator norm of an operator: $\ell^{\infty}(A) \rightarrow \ell^{\infty}(A)$.

Acknowledgement. One of us (C.A.) would like to thank Dr. H. Brézis for a useful suggestion.

\section{References}

1. Albanese, C., Fröhlich, J.: Periodic solutions of some infinite dimensional Hamiltonian systems associated with partial difference equations. Commun. Math. Phys. 116, 475-502 (1988) 
2. Rabinowitz, P.: Some global results for non-linear eigenvalue problems. J. Funct. Anal. 7, 487-513 (1971)

3. Albanese, C.: A continuation method for non-linear eigenvalue problem. J. Funct. Anal. (in press)

4. Albanese, C.: Localised solutions of Hartree equations for narrow band crystals. Commun. Math. Phys. (in press)

5. Wegner, F.: Bounds on the density of states for disordered systems. Z. Phys. B 22, 9 (1981)

6. Fröhlich, J., Spencer, T.: Absence of diffusion in the Anderson tight binding model for large disorder or low energy. Commun. Math. Phys. 88, 151 (1983)

7. Simon, B.: Correlation inequalities and the decay of correlations in ferromagnets. Commun. Math. Phys. 77, 111 (1980)

8. Chow, S.N., Hale, J.K.: Methods of bifurcation theory. Berlin, Heidelberg, New York: Springer 1982

Communicated by A. Jaffe

Received July 8, 1988 
